\title{
POSTERS AND DEMONSTRATIONS
}

\section{An Evaluation of JPEG and JPEG 2000 Irreversible Compression Algorithms Applied to Neurologic Computed Tomography and Magnetic Resonance Images}

\author{
V. Savcenko, B.J. Erickson, K.R. Persons, N.G. Campeau, J. Huston III, C.P. Wood, and S.A. Schreiner
}

We performed visual comparison of 200 head magnetic resonance (MR) and 200 head computed tomography (CT) images compressed at two levels using standard Joint Photographic Experts Group (JPEG) irreversible compression and a preliminary version of the JPEG $\mathbf{2 0 0 0}$ irreversible algorithm. Blinded evaluations by neuroradiologists compared original versus either JPEG or JPEG $\mathbf{2 0 0 0}$. We found that this version of JPEG $\mathbf{2 0 0 0}$ did not perform as well as the current JPEG for head CTs, but for MR images, JPEG 2000 performed as well or better. Around 7:1 compression ratio seemed to be a conservative point where there was no perceptible difference.

Copyright $\odot 2000$ by W.B. Saunders Company

$\mathbf{T}$

HE APPLICATION of a wavelet-based compression technique (JPEG 2000) is currently being developed by the Joint Photographic Experts Group (JPEG) committee, and will complement the discrete cosine transform approach used in current JPEG compression.

This study compared the effects of JPEG, ${ }^{1}$ and a preliminary version of the JPEG 2000 irreversible compression algorithms, on magnetic resonance (MR) and computed tomography (CT) images of the head. The objectives were to determine how much compression is acceptable for diagnostic purposes, and to compare the two algorithms.

\section{MATERIALS AND METHODS}

Prior to applying JPEG compression, a small subset of images was evaluated to select a "conservative" and a "borderline" quality factor that would be used for the study. Quality factors of

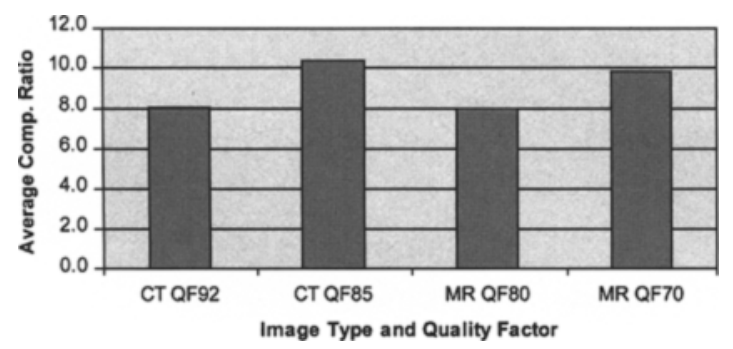

Fig 1. Average JPEG compression ratio by image type and quality factor for $200 \mathrm{MR}$ and $200 \mathrm{CT}$ images.

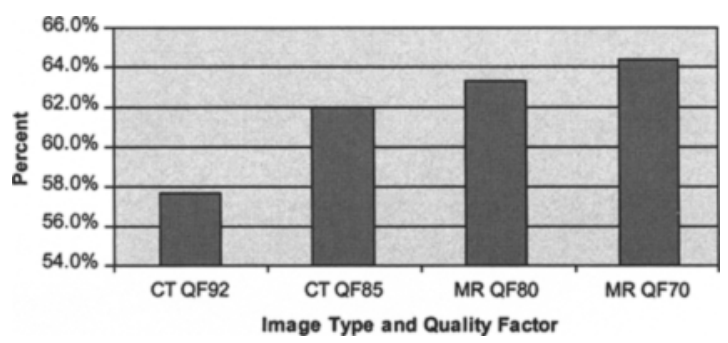

Fig 2. Percent of pairs where the original image was preferred over the JPEG compressed image as a function of image type and quality factor.

92/85 and 80/70 were selected as the "conservative"/"borderline" quality factors for CT and MR, respectively.

Initially, $200 \mathrm{CT}$ and $200 \mathrm{MR}$ images were each compressed at the two selected quality factors using the Independent JPEG group's JPEG algorithm. A double-blind protocol was used, where the original and compressed images were presented in random pairs to radiologists on a Digital Imaging and Communications in Medicine (DICOM) workstation. For each image pair, five board-certified neuroradiologists were asked to indicate their preference for the right or left image, and the magnitude of preference.

Subsequently, the same images were compressed and decompressed using an evaluation version of the JPEG 2000 compression algorithm (verification model 5.2, floating point). To compare effectiveness of the two compression algorithms, each image was compressed with JPEG 2000 to match (as closely as possible) the compression ratio that JPEG produced at each quality factor for that image. Ninety-three percent of the MR images (with a compression ratio $<12$ ), and $52 \%$ of $\mathrm{CT}$ images (with a compression ratio $<14$ ) matched the original JPEG compression ratio, with a maximum difference of 0.2 . The JPEG 2000 compressed image pairs were evaluated using the same five board-certified neuroradiologists, and double-blind protocol that were used in the first evaluation.

From the Departments of Diagnostic Radiology and Information Services, Mayo Clinic, Rochester, MN.

Address reprint requests to Bradley J. Erickson, MD, PhD, Diagnostic Radiology, Mayo Clinic Building., E2, 200 lst St SW, Rochester, MN 55905.E-mail: bje@mayo.edu.

Copyright (C) 2000 by W.B. Saunders Company

0897-1889/00/1302-1044\$10.00/0

doi:10.1053/jdim.2000.6874 


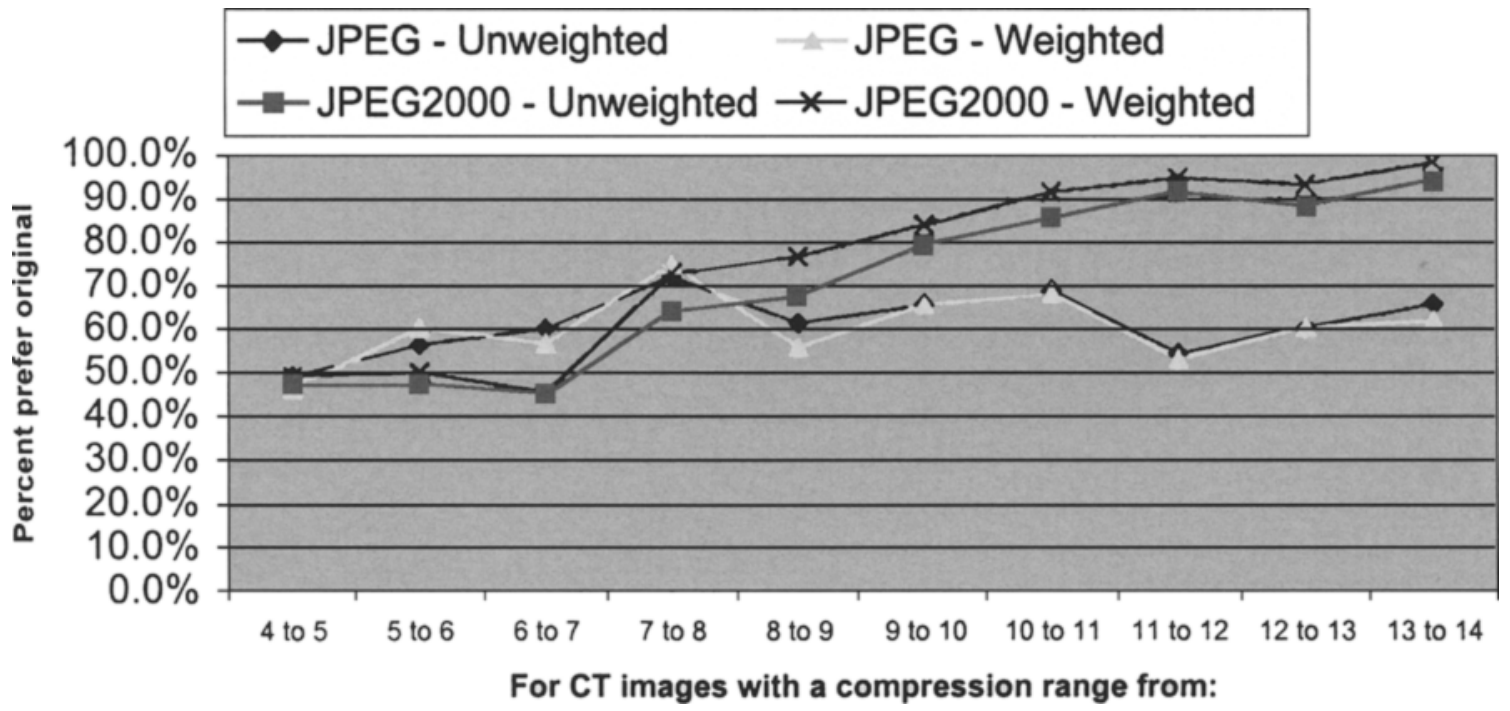

Fig 3. For those CT images where the JPEG and JPEG 2000 algorithms yielded compressed images with a compression ratio difference of $<0.2$, the percent of times when the original was preferred over the compressed as a function of the compression ratio range. The JPEG 2000 compression algorithm did not perform as well as JPEG for CT images at compression ratios $>8$. Also, based on the weighted results, the radiologists had a high confidence level in correctly picking the original over the compressed image with JPEG 2000.

\section{RESULTS}

Applying JPEG compression at the quality factors selected yielded compression ratios ranging from 4 to 48 , with most images having a compression ratio between 5 and 14. Figure 1 shows the average compression ratio by image type and quality factor for $200 \mathrm{MR}$ and $200 \mathrm{CT}$ images. The compression ratio measured was the ratio of the original image (without header) to the compressed image (without header).

Figure 2 shows the percent of pairs where the original image was preferred over the JPEG compressed image as a function of image type and quality factor.

Figure 3 shows, for those CT images for which the JPEG and JPEG 2000 algorithms yielded

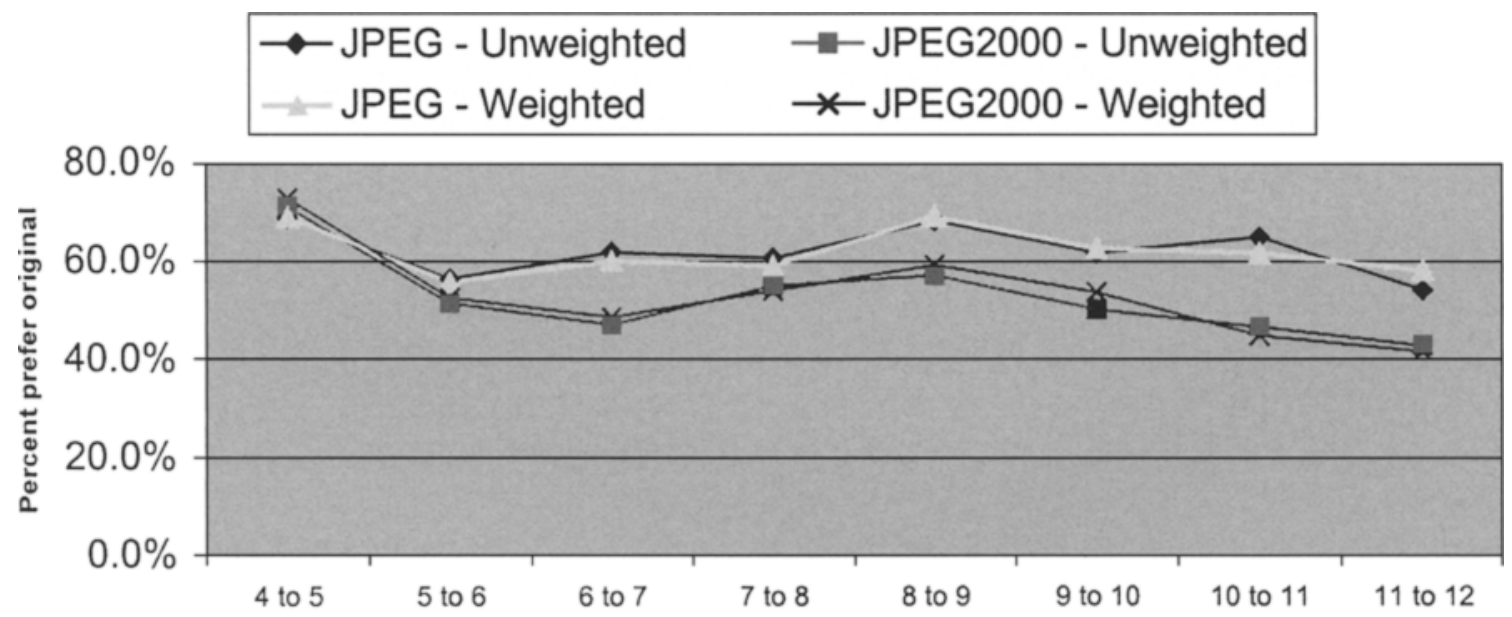

For MR images with a compression range from:

Fig 4. For those MR images where the JPEG and JPEG 2000 algorithms yielded compressed images with a compression ratio difference of $<\mathbf{0 . 2}$, the percent of times when the original was preferred over the compressed as a function of the compression ratio range. For MR images, the JPEG 2000 compression performed as well as or better than JPEG at all compression ratios measured. 
compressed images within a ratio of 0.2 of each other, the percent of time when the original was preferred over the compressed as a function of the compression ratio range. The JPEG 2000 compression algorithm did not perform as well as JPEG for $\mathrm{CT}$ images, especially at compression ratios between 8 and 14 .

Figure 4 shows, for those MR images where the JPEG and JPEG 2000 algorithms yielded compressed images within a ratio of 0.2 of each other, the percent of time when the original was preferred over the compressed as a function of the compression ratio range. For MR images, the JPEG 2000 compression algorithm performed as well as or better than JPEG at all compression ratios measured.

\section{CONCLUSIONS}

We found that JPEG irreversible compression could be applied to CT and MR images of the head at substantial compression ratios (7:1 or more) without significant perceptual degradation. We also found that while JPEG 2000, and its new wavelet methods, performed slightly better than the original discrete cosine transform (DCT)-based algorithm for MR images, it did not perform as well for CT images. We also note that neither quality factorbased specifications nor strict bit-rate specifications are acceptable methods for defining how much compression to apply to neurologic images.

\section{REFERENCE}

1. Wallace GK: The JPEG still picture compression standard. Comm ACM 34:30-44, 1991 